\title{
COHERENCE OF ONE POLYNOMIAL RING
}

\author{
WOLMER V. VASCONCELOS ${ }^{1}$
}

\begin{abstract}
The main result is that $A[x]$, the polynomial ring in one variable over the domain $A$ of global dimension two, is coherent. As an application, the quotient rings of $A[x]$ which have finite global dimension are examined.
\end{abstract}

Introduction. A ring is said to be coherent if all of its finitely generated ideals are finitely presented. Although supposedly a generalization of the noetherian condition the coherence property is, most often, a consequence of a variety of deeper properties of the ring. In $\S \S 1$ and 2 we will exemplify this by using the structure theory of a domain $A$ of global dimension two to prove that $A[x]$ is coherent. In $\S 3$ this result is applied to examine the rings of finite global dimension of the form $R[x] / I, I$ a finitely generated ideal.

1. Coherence of $A[x]:$ Local case. Let $A$ denote in this section a commutative local ring with maximal ideal $M$ and global dimension two (in particular every ideal of $A$ has projective dimension at most one).

We shall need the following description of $A$ [8], which together with [5] will be our source for terminology.

(1) $A$ is a coherent domain with G.C.D.

(2) If $M$ is principal or not finitely generated, $A$ is a valuation domain.

(3) Otherwise $M$ is generated by two elements, its other finitely generated prime ideals are principal and the remaining primes are linearly ordered and divisible by each prime element.

To resume: $A$ is either (i) a valuation domain, or (ii) a noetherian ring, or (iii) a hybrid of the preceding cases obtained in the following way. There is a valuation domain $V$ with maximal ideal $P$ and a noetherian regular local ring $N \subset V / P$ of dimension two with total ring of quotients $V / P$ and $A=f^{-1}(N)$ where $f$ is the canonical mapping $V \rightarrow V / P$. In this last case $A$ will be called an umbrella ring.

Presented to the Society, January 25, 1973; received by the editors January 19, 1973. AMS (MOS) subject classifications (1970). Primary 13B25; Secondary $13 \mathrm{C} 10$.

Key words and phrases. Projective dimension, global dimension, coherent ring, G.C.D. domain.

1 This research was partially supported by the National Science Foundation Grant GP-33133.

(c) Américan Mathematical Society 1973 
THEOREM 1.1. Let $A$ be a local ring of global dimension two; then $A[x]$ is coherent.

Proof. Let $B$ denote $A[x]$; we recall that $B$ is coherent if and only if $E=\Pi B$, a direct product of an arbitrary family of copies of $B$, is $B$-flat (cf. [3]). If $A$ is a valuation domain the result is an immediate consequence of $[7$, p. 25]. The noetherian case being trivial we shall assume, according to previous considerations, that $A$ is an umbrella ring.

To show the flatness of $E$ above we shall need the following technical result $[7$, p. 66].

Proposition 1.2. Let $R$ be a commutative ring, $S$ a multiplicative set of nonzero divisors of $R$ and let $E$ be an $R$-module. If (i) $E$ is $S$-torsion free, (ii) $E / s E$ is $R / s R$-flat for all elements in $S$ and, (iii) the localization $E_{S}$ is $R_{S^{-}}$ flat, then $E$ is $R$-flat.

In the above proposition let $R=A[x], A$ an umbrella ring and let $S$ be the multiplicative set generated by the prime elements of $A$. Notice that for each such element $s, A / s A$ is a noetherian ring and that $A_{S}$ is a valuation domain. Thus with $E$ the aforementioned direct product, the conditions (i) and (ii) of (1.2) are trivially satisfied and we proceed to test (iii). For that we will show that $\operatorname{Tor}^{B_{s}}\left(E_{S}, B_{S} / I\right)=0$ for each finitely generated ideal $I$ of $B_{S}$. Since $A_{S}$ is a valuation domain, $B_{S}$ is a G.C.D. domain (cf. [5, p. 42, Example 9]).

Let $I$ be a finitely generated ideal of $B_{S}$; if $I$ had a common factor, say $d$, we could write $I=d \cdot J$ and thus

$$
0 \rightarrow d B_{S} / I \rightarrow B_{S} / I \rightarrow B_{S} /(d) \rightarrow 0
$$

yields that $\operatorname{Tor}^{B_{s}}\left(E_{S}, B_{S} / I\right)=\operatorname{Tor}^{B_{s}}\left(E_{S}, B_{S} / J\right)$ as $E_{S}$ is torsion free.

Now let us assume $I$ generated by $n$ elements, with G.C.D. $=1$. Since $A_{S}$ is a valuation domain (and hence semihereditary) the weak global dimension of $A_{S}[x]$ is two [4]. As it is also coherent [7, p. 25] there is a resolution

$$
0 \rightarrow K \rightarrow B_{S}^{n} \rightarrow I \rightarrow 0
$$

with $K$ finitely generated and $B_{S}$-flat (and hence $B_{S}$-projective). But according to [11] $K$ is $B_{S}$-free of rank necessarily $n-1$.

Write the sequence above as

$$
0 \longrightarrow B_{S}^{n-1} \stackrel{f}{\longrightarrow} B_{S}^{n} \longrightarrow I \longrightarrow 0
$$

where by changing conveniently the generators of $I$ we may assume that the matrix representing $f$ has all of its entries in $A[x]$.

According to the well-known description of the ideals of projective dimension one presented as in $\left(^{*}\right)$ (cf. [2] or [5, p. 148, Example 8]), I 
can then be written $I=\left(d_{1}, \cdots, d_{n}\right)_{S}$, where the $d_{i}$ 's are the $(n-1) \times(n-1)$ minors of $f$. Let $D=\left(d_{1}, \cdots, d_{n}\right)$ be the corresponding ideal of $A[x]$. Write $d=$ G.C.D. of the $d_{i}$ 's (up to a unit) in $B$ (notice that $B$ is also a G.C.D. domain). After localizing in $B_{S}$ we conclude that $d$ becomes a unit in $B_{S}$ and thus may be taken to be an element of $S$.

We construct now the following commutative diagram with exact rows and column.

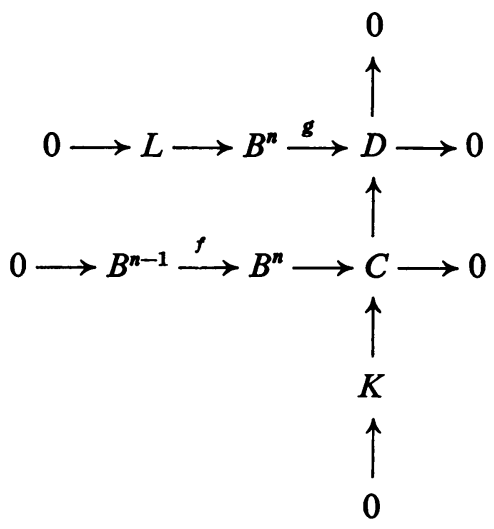

where $C$ is the cokernel of $f$ (no danger of confusion) and $L$ is the kernel of $g$ obtained by taking $\wedge^{n-1}\left({ }^{t} f\right)$ with the natural identifications (here $\Lambda$ denotes exterior power and $t$ the transpose). From the serpent lemma $[1$, p. 40] we get that $K \cong L / \operatorname{Image}(f)$. With the $d$ above

LEMMA 1.3. $d \cdot K=(0)$.

Proof. Let $\left(r_{1}, \cdots, r_{n}\right)$ be an element of $B^{n}$ such that $\sum r_{i} d_{i}=0$; it is enough to show that there exists $\left(x_{1}, \cdots, x_{n-1}\right)$ in $B^{n-1}$ such that

$$
\sum_{i=1}^{i=n} a_{j i} x_{i}=d r_{j}, \quad j=1, \cdots, n, \quad \text { where } f=\left(a_{j i}\right) .
$$

From Cramer's rule we have a solution $\left(x_{1}, \cdots, x_{n-1}\right)$ with components in the field of quotients of $B$, where, for instance,

$$
x_{1}=d_{1}^{-1} \cdot \operatorname{det}\left(\begin{array}{ll}
a_{2,2} \cdots a_{2, n-1}, & d r_{2} \\
a_{n, 2} \cdots a_{n, n-1}, & d r_{n}
\end{array}\right)
$$

so that $d_{1} x_{1}=d t_{1}$, with $t_{1} \in B$. Write $d_{1}=d e_{1}$ to get $e_{1} x_{1} \in B$; similarly we have $e_{j} x_{1} \in B$ for any $e_{j}\left(=d_{j} / d\right)$ so that $\left(e_{1}, \cdots, e_{n}\right) \cdot x_{1} \subset B$. As the ideal generated by the $e_{j}$ 's has 1 for its G.C.D. we conclude $x_{1} \in B$.

We are now in a position to conclude the proof of the theorem. For that 
tensor the diagram $\left({ }^{* *}\right)$ by $\cdot(\Pi B) \otimes_{A} A_{S}$ to obtain

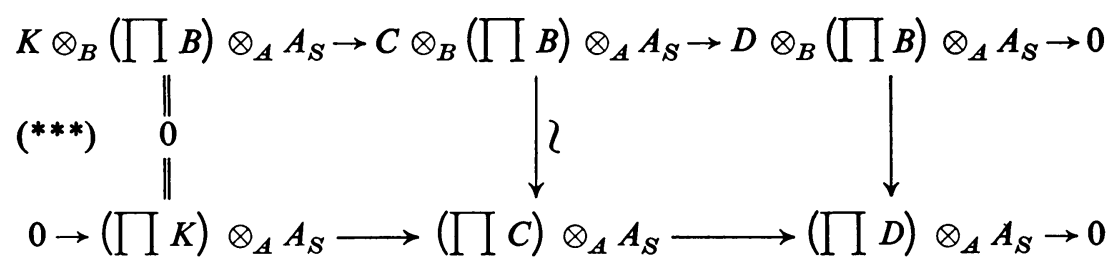

where the modules on the left are trivial by the lemma and the middle vertical map is an isomorphism since $C$ is finitely presented. This allows us to conclude that

$$
D \otimes_{B}\left(\prod B\right) \otimes_{A} A_{S} \hookrightarrow\left(\prod B\right) \otimes_{A} A_{S}
$$

which amounts to saying that $\operatorname{Tor}^{B_{s}}\left(E_{S}, B_{S} / I\right)=0$, as desired.

2. Coherence of $A[x]$ : Global case. In this section $A$ will be, unless otherwise specified, a domain of global dimension two. The proof that $A[x]$ is coherent will be a consequence of (i) the local case as discussed in the preceding section, (ii) the finite generation of certain prime ideals of $A$, and (iii) a theorem of Raynaud [7, Theorem 3.4.6].

The following result will initiate our discussion.

Proposition 2.1. Let A be a domain of global dimension two and let $M$ be a prime ideal containing two noncomparable prime ideals. Then $M$ is a finitely generated maximal ideal.

Before we embark on the proof notice that for each finitely generated ideal $I$ of $A$ we have an exact sequence

$$
0 \rightarrow L \rightarrow A^{n} \rightarrow I \rightarrow 0
$$

with $L A$-projective. As $A$ is a domain, it is easily seen by rank consideration that $L$ is finitely generated; hence $A$ is a coherent ring.

PROOF. From the local structure theory of [8] it follows that $M$ is a maximal ideal. Let $P, Q$ be two noncomparable prime ideals contained in $M$ and pick elements $a \in P \backslash Q, b \in Q \mid P$. Let $I$ be the ideal generated by $a$ and $b$. Resolve this ideal in the following way:

$$
0 \longrightarrow L \longrightarrow A^{2} \stackrel{\phi}{\longrightarrow}(a, b) \longrightarrow 0
$$

where $\phi(x, y)=a x-b y . L$ is then isomorphic to the ideal $(a): b=$ $\{r \in A \mid r b \in(a)\}$. Since $L$ is a projective module, we may pick, in the manner of $\left[1\right.$, p. 138], a partition of the unity $\left(f_{1}, \cdots, f_{n}\right)=A$ with the property that $L_{f}$ is $A_{f}$-free. (Here $f$ stands for any of the $f_{i}$ 's and $L_{f}$ is the module of fractions arising out of the multiplicative set of powers of $f$.) 
Clearly, in proving the finite generation of $M$ it is enough to consider only those localizations $A_{f}$ in which $M$ survives (i.e. $f \notin M$ ). Notice that in these cases the choices of $a, b$ are not affected. Thus change notation and assume that $L$ is a free $A$-module, say $L=A \cdot \alpha, \alpha=(u, v)$. As $(b, a) \in$ $L,(b, a)=c \cdot(u, v)$. It is easy to see that $c$ is the G.C.D. of $a, b$ (Note. This is not to say that $A$ is a G.C.D. domain!) and $u, v$ are elements in $M$ forming a regular sequence. Write now $J=(u, v)$ and apply twice the change of rings theorem of $[6, \mathrm{p} .172]$ to have that $A / J$ is a ring of finitistic projective dimension zero, i.e. $A / J$ is a perfect ring. According to $[3$, p. 467] however, $A / J$ being both perfect and coherent is artinian. In particular, $M / J$ is finitely generated and $M$ will also be finitely generated.

We shall refer to prime ideals of $A$ such as $M$ as a special maximal ideal.

For convenience, we quote the following remarkable result of Raynaud which will be the key to proving the coherence of $A[x]$. For simplicity we state it for the case of a domain.

THEOREM 2.2. Let $B$ be an algebra of finite presentation over the domain $A$ and let $E$ be a $B$-module of finite type. Then the set $U$ of primes of $B$ such that $E_{P}$ is $A_{p}$-flat $(p=$ inverse image of $P$ in $A)$ is an open subset of $\operatorname{Spec}(B)$ and $E(U)$ is a $B(U)$-module of finite presentation.

For our setting, let $A$ be a domain of global dimension two, let $B=$ $A\left[x_{1}, \cdots, x_{n}\right]$, the polynomial ring in $n$ variables over $A$ and let $E$ be a finitely generated ideal $I$ of $B$.

Proposition 2.3. In the above conditions, if $B_{p}$ is coherent for each prime $p$ of $A$ then $B$ is coherent.

Taking into account Theorem 1.1 we can state the main result of this paper.

Corollary 2.4. If $A$ is a domain of global dimension two; then $A[x]$ is coherent.

Proof of 2.3. Let $P$ be a prime ideal of $B$ and let $p=P \cap A$. If $I_{P}$ is not $A_{p}$-flat, $p$ must be a special maximal ideal as otherwise $A_{p}$ is a valuation domain [8]. In particular it follows that $V=\operatorname{Spec}(B) \backslash U$, in the notation of Theorem 2.2, is a closed set and the primes in it are finitely generated. Thus $V$ is a noetherian space and can be expressed as a finite union of its irreducible components

$$
V=V\left(P_{1}\right) \cup \cdots \cup V\left(P_{n}\right) .
$$

Write $\boldsymbol{p}_{i}=A \cap \boldsymbol{P}_{i}$ and let $J=\boldsymbol{p}_{1} \cdots \boldsymbol{p}_{n} ; J$ is a finitely generated ideal of $A$, say $J=\left(f_{1}, \cdots, f_{m}\right)\left(f_{i} \neq 0\right)$. Consider the following change of rings

$$
B \rightarrow B_{p_{1}} \times \cdots \times B_{p_{n}} \times B_{f_{1}} \times \cdots \times B_{f_{m}} ;
$$


in a component such as $B_{p_{i}}$ the ideal $I B_{p_{i}}$ is of finite presentation by hypothesis, while $I B_{f_{i}}$ is $A_{f_{j}}$-flat by construction and thus of finite presentation by the second part of Theorem 2.2. Finally, notice that the change of rings is faithfully flat and the conclusion follows.

3. Regular quotients of $A[x]$. Let $A$ be a domain of global dimension two and let $I$ be a finitely generated ideal of $A[x]$. Using the results of [8] and that of the preceding section we study some of the regular (i.e. of finite global dimension) rings of the type $C=A[x] / I$. The main intent is to study the minimal prime ideals of $C$ as its finiteness is closely related to the noetherianness of $C$. To make the arguments much simpler we will only consider the local case.

This analysis will depend, firstly, on the possible projective dimension of $I(0,1$, and 2 , yielding correspondingly three distinct cases). An initial remark to make is that since $A[x]$ is a G.C.D. domain, if we write $I=d \cdot J$ with $d=\mathrm{G}$.C.D. of $I$, then $(d, J)=(1)$. Indeed, otherwise for some maximal ideal $M, C_{M}$ would be coherent by the preceding section, regular but not a domain, which conflicts with [10]. More generally we have that any factorization of $I$ yields a decomposition of $C$ into a direct product.

Case 0. $I$ is a projective ideal. Then $I=(f)$, a polynomial. Let $d$ be the G.C.D. of the coefficients of $f, f=d \cdot g$. We may also factor $g$ into $g=g_{1} \cdots g_{n}$ where each $g_{i}$ is a prime element. Reasoning as before we conclude

$$
C=(A /(d))[x] \times A[x] /\left(g_{1}\right) \times \cdots \times A[x] /\left(g_{n}\right) .
$$

Notice that $d$ (if not trivial) is an indecomposable element of $A$ and, this ring being a G.C.D., $d$ is a prime element. Taking into account the structure of $A$ we may even add that $d$ is an element in $M \backslash M^{2}$, and that this component of $C$ is noetherian.

By the change of rings theorem of $[5$, p. 124, Theorem C] each of the other components is a domain of global dimension at most two.

Case 1. Projective dimension of $I=1$. As before write $I=f \cdot J, f=$ G.C.D. of $I$, to get

$$
C=A[x] /(f) \times A[x] / J .
$$

The component $A[x] /(f)$ is as described in the previous case while $A[x] / J$ is, by the change of rings theorem of [9], a hereditary ring, hopefully a Dedekind ring (i.e. a finite direct product of Dedekind domains).

We show that this last statement is correct, that is $A[x] / J$ is noetherian, whenever the Krull dimension of $A$ is finite. Note that $J \cap A=L \neq(0)$ as otherwise there is $0 \neq b \in A$ such that $b \cdot J \subset(g)$. $J$ being a radical ideal, $L$ is also a radical ideal. Confronting this remark with the possible nature of $A$ we get the following. If $A$ is a valuation ring, $L$ is a prime ideal; if 
$A$ is an umbrella ring but $A / L$ is not noetherian, $L$ is also a prime ideal. These are the only cases for which we must show the noetherianness of $A[x] / J$. With $J^{\prime}$ denoting the image of $J$ in $(A / L)[x]$ we may write our ring as $(A / L)[x] / J^{\prime}$ and $J^{\prime} \cap(A / L)=(0)$.

Proposition 3.1. Let $A$ be a local ring of global dimension two and let $L$ be a prime ideal not finitely generated. Then $A / L$ has global dimension at most two.

Proof. We are thus avoiding the noetherian case. Let $I$ be an ideal properly containing $L$. The sequence

yields

$$
0 \rightarrow I \rightarrow A \rightarrow A / I \rightarrow 0
$$

$$
0 \rightarrow L / L I \rightarrow I / L I \rightarrow A / L \rightarrow A / I \rightarrow 0 .
$$

If we show $L=L I$ (i.e. $\operatorname{Tor}_{1}^{A}(A / I, A / L)=0$ ) and taking into account that $L$ is $A$-flat it will follow that an $A / L$-resolution for $A / I$ may be obtained by tensoring an $A$-resolution of $A / I$ by $A / L$.

First consider the case of a finitely generated ideal $I=\left(a_{1}, \cdots, a_{n}\right)=$ $d\left(b_{1}, \cdots, b_{n}\right)$ not contained in $L$ (here $d=$ G.C.D. of the $a_{i}$ 's). As $\left(b_{1}, \cdots, b_{n}\right)$ contains a power of the maximal ideal $M$ of $A$ (we are really examining only the umbrella case [7]) and $L M=L, L \cap d\left(b_{1}, \cdots, b_{n}\right)=d L$, that is $I \cap L=L I$.

For an arbitrary ideal $I$ properly containing $L$, write $I=\sum I_{\alpha}, I_{\alpha}$ running over the finitely generated subideals of $I$ not contained in $L$. Then $I \cap L=$ $\sum L \cap I_{\alpha}=\sum L I_{\alpha}=L I$ and thus $L=L I$ as desired.

To complete our examination of Case 1, picking up the notation of before we have $C=A[x] /(f) \times A^{\prime}[x] / J^{\prime}$ and the conclusion follows as the second component is hereditary of lower Krull dimension.

REMARK. It is likely that in all cases (even in the missing one, that of projective dimension two) one always obtains that $C$ has only a finite number of idempotents.

\section{REFERENCES}

1. N. Bourbaki, Eléments de mathématique. Fasc. XXVII. Algebre commutative. Chap. 1: Modules plats. Chap. 2: Localisation, Actualités Sci. Indust., no. 1290, Hermann, Paris, 1961. MR 36 \#146.

2. L. Burch, On ideals of finite homological dimension in local rings, Proc. Cambridge Philos. Soc. 64 (1968), 941-948. MR 37 \#5208.

3. S. U. Chase, Direct product of modules, Trans. Amer. Math. Soc. 97 (1960), 457-473. MR 22 \#11017.

4. C. U. Jensen, On homological dimensions of rings with countably generated ideals, Math. Scand. 18 (1966), 97-105. MR 34 \#7611.

5. I. Kaplansky, Commutative rings, Allyn and Bacon, Boston, Mass., 1970. MR 40 \#7234. 
6. I. Kaplansky, Fields and rings, University of Chicago Press, Chicago, Ill., 1969. MR 42 \#4345.

7. M. Raynaud and L. Gruson, Critères de platitude et de projectivité, Invent. Math. 13 (1971), 1-89.

8. W. V. Vasconcelos, The local rings of global dimension two, Proc. Amer. Math. Soc. 35 (1972), 381-386.

9. - Rings of global dimension two, Proc. Comm. Algebra Conf. (Lawrence, Kansas, 1972), Lecture Notes in Math., no. 311, Springer-Verlag, Berlin, 1973.

10. —_, Finiteness in projective ideals, J. Algebra 25 (1973), 269-278.

11. W: V. Vasconcelos and A. Simis, Projective modules over $R[x], R$ valuation ring, are free, Notices Amer. Math. Soc. 18 (1971), 805. Abstract \#71T-A178.

Department of Mathematics, Rutgers University, New Brunswick, New JERSEY 08903 